\title{
Hint1 expression inhibits proliferation and promotes radiosensitivity of human SGC7901 gastric cancer cells
}

\author{
XIAOWEI WEI ${ }^{1}$, JIN ZHOU ${ }^{2}$, LINGZHI HONG ${ }^{1}$, ZHI XU ${ }^{1}$, HUANYU ZHAO $^{1}$, XIAOMIN WU ${ }^{1}$ and JINFEI CHEN ${ }^{1}$ \\ Departments of ${ }^{1}$ Oncology and ${ }^{2}$ General Surgery, Nanjing First Hospital, \\ Nanjing Medical University, Nanjing, Jiangsu 210006, P.R. China
}

Received October 8, 2015; Accepted March 21, 2017

DOI: $10.3892 / \mathrm{ol} .2018 .8900$

\begin{abstract}
Gastric cancer is a prevalent, malignant tumor that frequently escapes treatment. Histidine triad nucleotide-binding protein 1 (Hint1) is a haploinsufficient tumor suppressor gene which contributes to intercellular communication, helps to regulate cell proliferation and survival, and is frequently underexpressed in gastric cancer. To examine the involvement of Hint1 in gastric cancer, small interfering RNA was used to knock down Hint1 expression in the human gastric cancer cell line SGC-7901. The data revealed that Hint1 inhibited cell proliferation, reduced radiation-induced DNA damage repair and caused G1 phase arrest, which increased the radiosensitivity of gastric cancer cells. Further mechanistic studies revealed a novel function of Hint1, whereby it acted as a negative regulator of extracellular signal-regulated kinase. These results demonstrated the critical function of Hint1 in the biology of human gastric cancer. Acting as a tumor growth suppressor and a radiosensitive agent, this protein is a potential biomarker and may be an attractive target for specific therapeutic interventions against gastric cancer.
\end{abstract}

\section{Introduction}

Gastric cancer (GC) is the most prevalent digestive system carcinoma (1). In 2012, it was diagnosed in 950,000 patients and caused 723,000 mortalities (1). Its malignance is due to its capacity for rapid proliferation and resistance to chemoradiotherapy. While the biological events and key signaling pathway disruptions that drive its growth are being studied at present, the underlying mechanisms of resistance to conventional DNA-damaging agents and ionizing radiation (IR) remain largely unknown.

Correspondence to: Dr Jinfei Chen, Department of Oncology, Nanjing First Hospital, Nanjing Medical University, 68 Changle Road, Nanjing, Jiangsu 210006, P.R. China

E-mail: jinfeichen@sohu.com

Key words: histidine triad nucleotide binding protein 1, gastric cancer, proliferation, radiosensitivity, extracellular signal-regulated kinases
Histidine triad nucleotide binding protein 1 (Hint1) is a haploinsufficient tumor suppressor gene which contributes to intercellular communication, helps to regulate cell proliferation and survival, and is frequently underexpressed in the early stages of oncogenesis (2-5). Hint1 knockout mice demonstrated a marked increase in susceptibility to colorectal, mammary and ovarian tumors $(2,3)$. In 2011, our team first reported that Hint1 was underexpressed in human GC tissues at the protein and gene level. Its downregulation was associated with poor tumor cell differentiation and bacterial or viral infection, including infection by Helicobacter pylori or the Epstein-Barr virus, suggesting patients with Hint1 underexpression may present with biologically aggressive tumors and poor prognosis (6).

The tumor suppressive effects of Hint1 protein primarily serve an inhibitory function in a number of gene transcription control pathways. For instance, Hint1 promotes apoptosis via upregulation of p53 and downregulation of B cell lymphoma-2 in the SW480 human colon cancer cell line and the MCF7 breast cancer cell line (7). Upon association with the plenty of SH3 domains protein and mitogen activated protein kinase 9 complex, Hint1 inhibits activity of the activator protein-1 transcription factor responsible for the proliferation and angiogenesis of colon cancer cells (8). In addition, Hint1 enhances cellular responses to DNA damage by regulating the functions of $\gamma$-H2A histone family member X and ATM serine/threonine kinase (ATM) in normal cells (9). However, little is known regarding the effect of Hintl on radiotherapy in cancer, even though a relationship between Hint1 and DNA damage repair was reported previously (9).

The present study analyzed the tumor suppressive effects of Hint1 in the SGC7901 gastric cancer cell line. Its inhibition of cell viability in this cell line was demonstrated, and the involved signaling cascades were investigated. Hint1 may negatively regulate extracellular signal-regulated kinase (ERK), which is involved in gastric carcinogenesis. In addition, Hint1 prevented IR-induced DNA damage repair in SGC7901 cells via the repression of Cyclin D1-dependent retinoblastoma protein ( $\mathrm{Rb}$ protein) phosphorylation, which induced G1 arrest and cell death.

\section{Materials and methods}

Cell culture and treatment. The SGC7901 and AGS human gastric cancer cell lines were obtained from the Cell Bank of 
Type Culture Collection of the Chinese Academy of Sciences (Shanghai, China). Cells were maintained in RPMI-1640 medium (Invitrogen; Thermo Fisher Scientific, Inc., Waltham, MA, USA), supplemented with $10 \%$ fetal bovine serum (Gibco; Thermo Fisher Scientific, Inc.), and incubated in a $100 \%$ moist incubator with $5 \% \mathrm{CO}_{2}$ at $37^{\circ} \mathrm{C}$. A Varian medical linear accelerator (Varian Medical Systems, Palo Alto, CA, USA), offered by the Department of Oncology, Nanjing First Hospital (Nanjing, China) was used to treat the cells. Gastric cancer cells were plated at a density of $1 \times 10^{4}$ cells/well into 96-well plates, and were incubated overnight. Then, cells were treated with $0,1,2,4$ or 6 Gy X-ray or $50 \mu \mathrm{M}$ PD98059 for $24 \mathrm{~h}$ at $37^{\circ} \mathrm{C}$ (Sigma-Aldrich; Merck KGaA, Darmstadt, Germany), a specific ERK inhibitor (ERKi), for further experiments.

RNA interference. RNA interference was used to selectively knock down Hint1 in SGC7901 cells. The sequence of pGPU6/green fluorescent protein/Neo-short hairpin RNA (shRNA)-Hint1 (Sangon Biotech Co., Ltd., Shanghai, China) was 5'-CCGGCGACACGATCTTTGGGAAGATCTCGA GATCTTCCCAAAGATCGTGTCGTTTTTG-3'. Cells were transfected with the aforementioned shRNA using Lipofectamine 2000 (Invitrogen; Thermo Fisher Scientific, Inc.) for 24-72 h. Then cells were re-seeded in RPMI-1640 medium containing G418 (400 $\mu \mathrm{g} / \mathrm{ml}$; Gibco; Thermo Fisher Scientific, Inc.) to enrich the culture for cells that were successfully transfected. Following $120 \mathrm{~h}$ transfection, the cells were harvested to determine knockdown efficiency by reverse transcription-quantitative polymerase chain reaction (RT-qPCR) and western blot analysis. A non-targeting shRNA vector (cat. no. E-07/F-07; Shanghai GenePharma Co., Ltd., Shanghai, China.) was used as a negative control for all experiments.

Colony survival assay. A colony survival assay was performed to determine the influence of Hint1 on SGC7901 cell proliferation. Exponentially growing cells were seeded at a low density in a 6-well plate $(80$ cells/well plate; Corning Incorporated, Corning, NY, USA) and allowed to grow for 7-10 days in RPMI-1640 medium. The media was then removed and replaced with $0.1 \%$ crystal violet dye. The size of live colonies which contained $>50$ cells was evaluated using a fluorescence microscope (Olympus IX71; Olympus Corporation, Tokyo, Japan) at magnification, $x 200$. The number of colonies were then counted and the proliferation ratio was calculated as the ratio of the number of colonies in Hint1 silenced cells formed to those formed by the vector control group.

MTT assay. SGC7901 and AGS cells $\left(1 \times 10^{4}\right)$ were seeded in 96-well plates and then treated with $0,1,2,4$ or 6 Gy X-ray or PD98059 $(50 \mu \mathrm{m})$. MTT reagent (10 $\mu \mathrm{l} /$ well; Trivegen, Gaithersburg, MD, USA) was added and cells were left inside the incubator for a further $4 \mathrm{~h}$ at $37^{\circ} \mathrm{C}$, followed by the addition of $100 \mu \mathrm{l}$ detergent reagent (Trivegen) according to the manufacturer's protocol. Absorbance of the colored solution was measured by a fully automated multi-detection microplate reader (POLARstar Optima; BMG Labtech GmbH, Ortenberg, Germany) at $570 \mathrm{~nm}$.

Comet assay. The alkaline comet assay was performed in SGC7901 cells either directly following irradiation or following a recovery of $24 \mathrm{~h}$ in RPMI-1640 medium supplemented with $10 \%$ fetal bovine serum using the OxiSelect Comet Assay kit (Cell Biolabs, Inc., San Diego, CA, USA). All steps were performed on ice or in the cold to minimize repair processes. Images were recorded using a fluorescence microscope (Olympus IX71; Olympus Corporation, Tokyo, Japan) at magnification, $\mathrm{x} 200$. For each data point, 2-3 areas on parallel slides were scored with 51-60 cells each, and DNA in tail (\%) was calculated for each cell using the image-analysis software CaspLab (version 1.2.3 beta1; http://casplab.com/download). The median of DNA in tail (\%) was calculated for each area, and the presented values are the means of the medians of each data point.

Protein extraction and western blot. A total of $1 \times 10^{7}$ SGC7901 or AGS cells were collected and lysed using ice-cold lysis buffer (50 mM Tris, $\mathrm{pH} 7.4,150 \mathrm{mM} \mathrm{NaCl}$, $1 \%$ SDS, $1 \mathrm{mM}$ EDTA, 1\% NP-40) containing $1 \mathrm{mM}$ protein inhibitor and $1 \mathrm{mM}$ phenylmethylsulfonyl fluoride for $30 \mathrm{~min}$ on ice. The lysates were centrifuged at $10,000 \mathrm{x} \mathrm{g}$ at $4^{\circ} \mathrm{C}$ for $10 \mathrm{~min}$ and the supernatants were collected. Protein concentration was measured using a bicinchoninic acid protein assay (Pierce; Thermo Fisher Scientific, Inc.). Equal amounts of $30 \mu \mathrm{g}$ total protein for each sample was loaded and separated by 7.5-12.5\% SDS-PAGE and then transferred onto polyvinylidene difluoride membranes (Amersham Biosciences). Membranes were blocked with $5 \%$ skim milk in TBST (20 mM Tris- $\mathrm{HCl}, \mathrm{pH} 7.4,150 \mathrm{mM}$ $\mathrm{NaCl}, 0.1 \%$ Tween-20) for $1 \mathrm{~h}$ and incubated with specific antibodies diluted as needed at $4^{\circ} \mathrm{C}$ overnight. $\beta$-actin was used as the loading control. Then, membranes were incubated with goat anti-rabbit IgG (cat. no. sc-2040; 1:400) or goat anti-mouse IgG (cat no. sc-2039; 1:400; both Santa Cruz Biotechnology, Inc., Dallas, TX, USA) secondary antibodies for $1 \mathrm{~h}$ at room temperature. Target proteins were visualized with an Amersham Enhanced Chemiluminesence Western Blotting Detection kit following the manufacturer's protocol. (cat. no. RPN2108, GE Healthcare Life Sciences, Chalfont, UK). The primary antibodies included anti-Hint1 (1:500; ab64071; Abcam, Cambridge, UK), anti-phosphorylated (p-)ERK1/2 (1:250; cat. no. 4370), anti-ERK1/2 (1:1,000; cat. no. 9107; Cell Signaling Technology, Inc., Danvers,

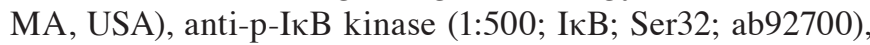
anti-nuclear factor- $\kappa \mathrm{B}(\mathrm{NF}-\kappa \mathrm{B})$ p65 (1:1,000; Ser536; cat no. ab86299), anti-ATM (1:300; cat no. ab17995; all Abcam, Cambridge, UK), anti-p-ATM (1:1,000; Ser1981; cat. no. 13050), anti-p-ATR serine/threonine protein kinase (1:500; ATR; Ser428; cat. no. 2853), anti-ATR (1:500; cat. no. 2790; all Cell Signaling Technology, Inc.), anti-Cyclin D1 (1:500; ab137875; Abcam), anti-p-Rb (1:1,000; Ser780; cat. no. 5225), anti-BCL2 associated X, apoptosis regulator (1:1,000; Bax; cat. no. 2772; both Cell Signaling Technology, Inc.) and anti- $\beta$-actin antibody (1:1,000; A1978, Sigma-Aldrich; Merck KGaA).

$R T$-qPCR. Hint1 mRNA levels were determined in SGC7901 cells using RT-qPCR. Total RNA was extracted from cells using an RNeasy kit (Qiagen, Inc., Valencia, CA, USA) and RT-qPCR was performed using a TaqMan reverse transcription kit (Thermo Fisher Scientific, Inc.) according to the 


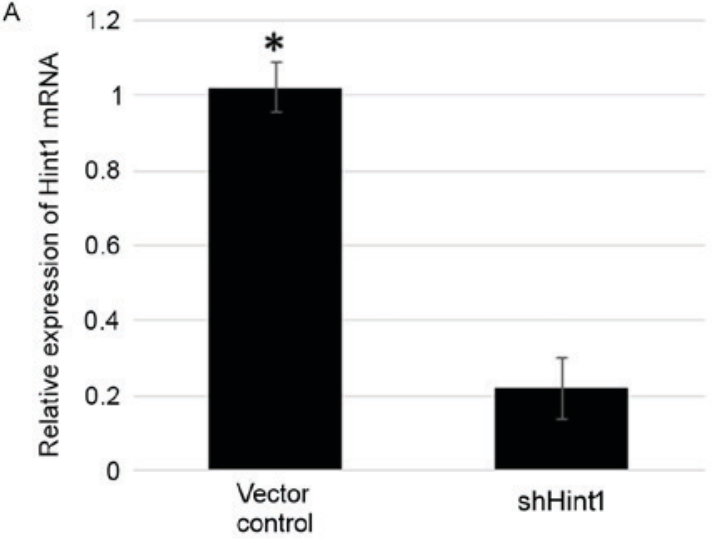

C

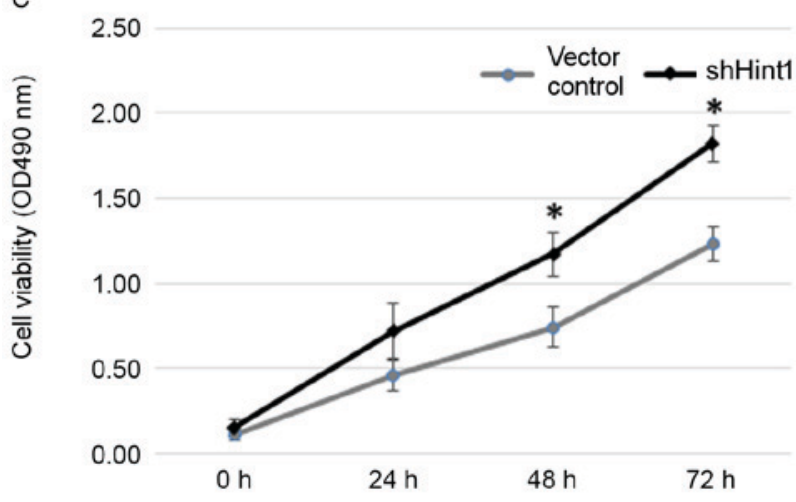

B

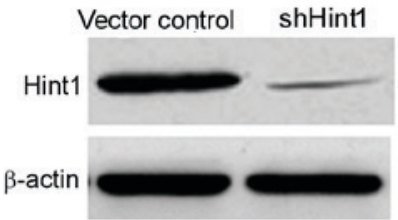

D

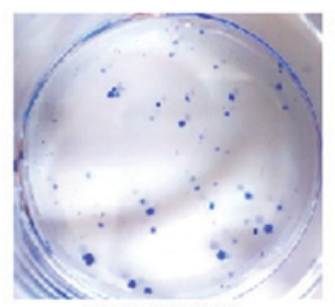

Vector control

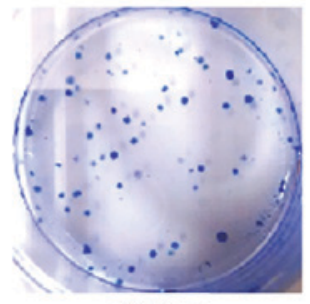

shHint1

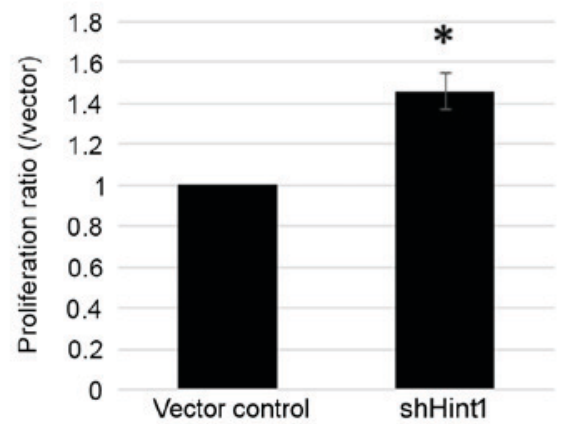

Figure 1. Effect of transfection with shHint1 on Hint1 mRNA and protein expression levels, and SGC7901 cell viability and proliferation. (A) Reverse transcription-quantitative polymerase chain reaction was performed to measure Hint1 mRNA expression in control vector cells and shHint1 cells. (B) Western blot analysis was performed to measure Hint1 protein expression in control vector cells and shHint1 cells. (C) Cell viability was assessed by MTT assay. (D) Cell proliferation ratio was determined by colony survival assay. Data are presented as the mean \pm standard error of the mean. * $\mathrm{P}<0.05$ vs. vector control. si, small interfering RNA; Hint1, histidine triad nucleotide-binding protein 1.

manufacturer's protocol. The PCR primers were as follows: Human Hint1 forward, 5'-ATTTCCCCTCAAGCACCA ACA-3' and reverse, 5'-ATCAGCAGCACATTTCTTGCC-3'; $\beta$-actin forward, 5'-CCCATCTATGAGGGTTACGC-3' and reverse, 5'-TTTAATGTCACGCACGATTTC-3' (Invitrogen; Thermo Fisher Scientific, Inc.). The thermocycler conditions were as follows: Initial denaturation at $95^{\circ} \mathrm{C}$ for $15 \mathrm{sec}$, then 40 cycles at $95^{\circ} \mathrm{C}$ for $5 \mathrm{sec}$ and $60^{\circ} \mathrm{C}$ for $30 \mathrm{sec}$. Relative expression to the control gene was determined using the $\Delta \Delta \mathrm{Cq}$ method (10). The experiments were repeated three times.

$N F-\kappa B$ transcription factor DNA binding activity. SGC7901 cells were treated as described previously. Nuclear extracts were prepared by a nuclear extract kit (Active Motif, Carlsbad, CA, USA) and subsequently analyzed for $\mathrm{NF}-\kappa \mathrm{B}$ activity using the NF- $\kappa$ B p65 Transcription Factor Assay kit (Cayman Chemical Company, Ann Arbor, MI, USA) according to the manufacturer's protocol. Briefly, cellular nuclear extracts were purified and plated in a 96-well plate. Then, samples were incubated with $\mathrm{NF}-\kappa \mathrm{B}$ (p65) primary antibody and secondary antibody successively. Absorbance of each well measured by a fully automated multi-detection microplate reader (POLARstar OPTIMA) at $450 \mathrm{~nm}$.

Flow cytometric analysis of the cell cycle. SGC7901 cells $\left(2 \times 10^{5}\right)$ were seeded in 6-well plates with RPMI-1640 medium supplemented with $10 \%$ fetal bovine serum and then irradiated with X-rays (6 Gy). The cells were harvested either prior to irradiation or following a recovery period of $24 \mathrm{~h}$. Then, cells were washed three times with cold PBS and resuspended in $1 \mathrm{ml}$ staining solution $(50 \mu \mathrm{g} / \mathrm{ml}$ propidium iodide, $20 \mu \mathrm{g} / \mathrm{ml}$ RNase A). Following incubation for $30 \mathrm{~min}$ at room temperature, samples were analyzed using a fluorescence-activated cell sorting flow cytometer (BD Biosciences, Franklin Lakes, NJ, USA). The percentage of cells in G0/G1, S, and G2-M phases was calculated and compared with ModFit LT software (version 3.2; Verity Software House, Topsham, ME, USA).

Statistical analysis. All experiments were performed at least in triplicate on separate experimental days. Statistical differences between the values obtained in different experimental settings were evaluated by the means of analysis of variance (two-way or one-way, as appropriate) with post hoc Duncan's range tests, or unpaired Student's t-tests, using IBM SPSS 21.0 software (IBM SPSS, Armonk, NY, USA) for Windows. P<0.05 was considered to indicate a statistically significant difference.

\section{Results}

Growth inhibitory effects of Hintl in SGC7901 cells. To analyze the involvement of Hint1 in gastric cancer, a shRNA-Hint1 vector was transfected into SGC7901 gastric 


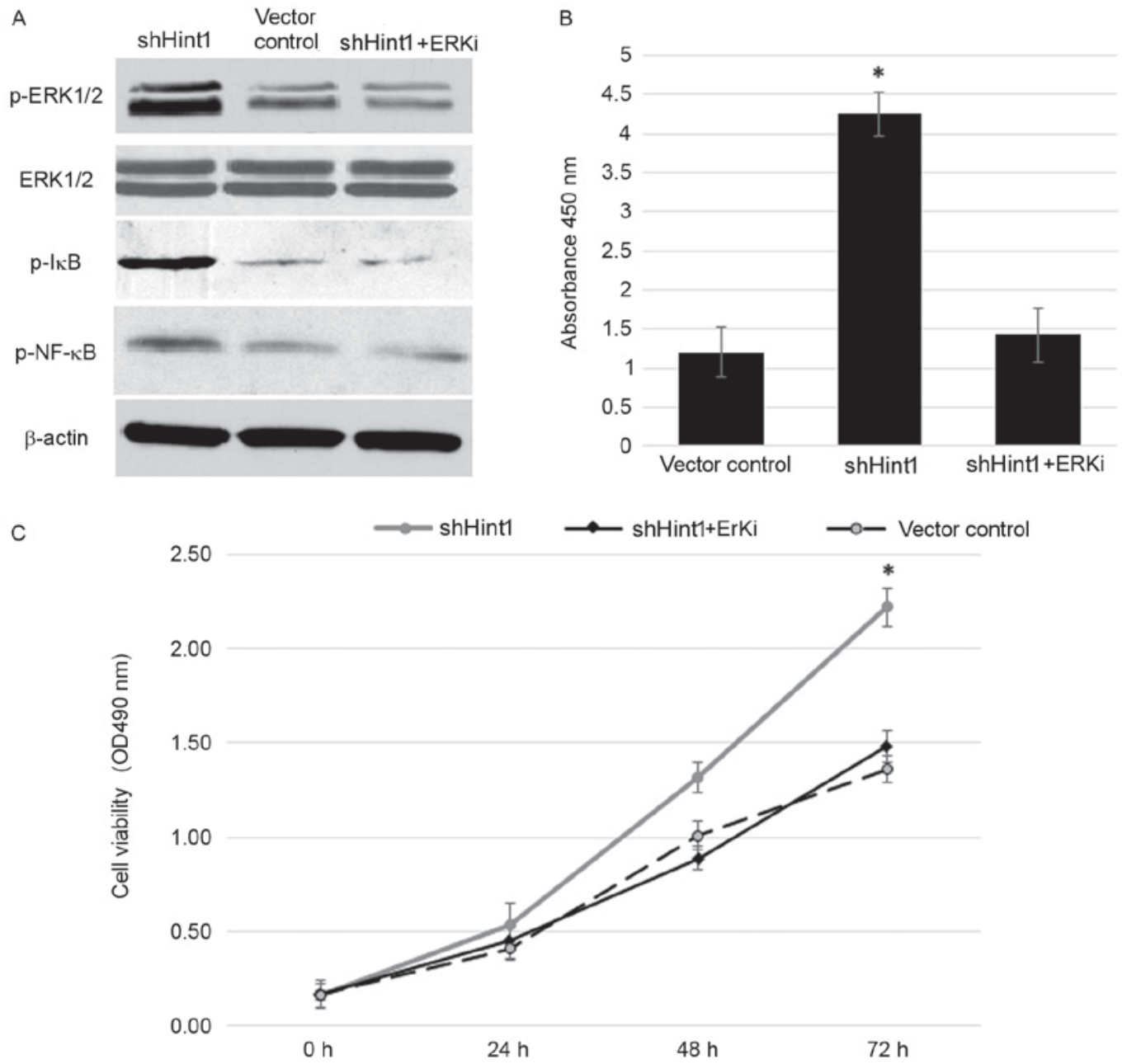

Figure 2. Effect of Hint1 interference on the ERK signaling pathway. (A) Western blot analysis was performed to measure ERK, IкB and NF- $\kappa B$ protein

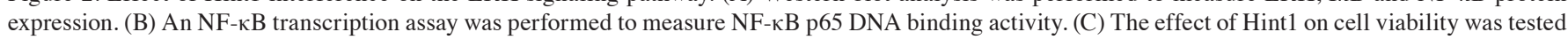
by MTT assay. "P<0.05 vs. vector control and shHint1+ERKi groups. Hint1, histidine triad nucleotide-binding protein 1; ERK, extracellular signal-regulated

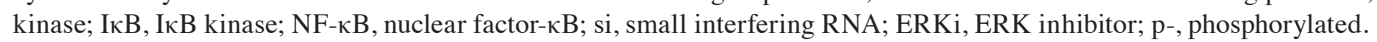

cancer cells. Transfection with the shHint1 vector resulted in significantly reduced Hint1 mRNA expression and visibly reduced Hint1 protein expression (Fig. 1A and B, respectively). In the subsequent MTT assay, cells transfected with shHint1 demonstrated significantly increased viability compared with the vector control group (Fig. 1C). Meanwhile, a colony survival assay was performed to verify the results of this test. There was a $\sim 1.5$ fold increase in the number of colonies observed in the shHint1 group compared with the vector control under the same culture conditions and time (Fig. 1D). These data demonstrated an inhibitory function of Hint1 on gastric cancer cell viability.

Suppression of ERK-dependent $N F-\kappa B$ activation is involved in Hint1-mediated retardation of cell viability. The ERK signaling cascade is a central mitogen-activated protein kinase (MAPK) pathway that regulates various oncogenic responses, including proliferation and survival $(11,12)$. To further investigate the mechanisms by which Hint1 inhibited the viability of gastric cancer cells, the interactions between Hint1 and ERK were determined. The results revealed that ERK phosphorylation activity was significantly increased following knockdown of Hint1 in SCG7901 cells (Fig. 2A).
Activated ERK subsequently induced I $\kappa$ B phosphorylation and activated the NF- $\mathrm{BB}$ p65 signal pathway when lacking regulation from Hint1 (Fig. 2A and B). These cascades were repressed by ERKi, demonstrating that the activation of NF- $\mathrm{KB}$ p65 following Hint1 knockdown was ERK dependent. To verify this result, the effect on cell viability in the shHint1 group was analyzed following treatment with ERKi. ERKi efficiently reversed the increased viability induced by Hint 1 deficiency (Fig. 2C). These data indicated that Hintl may be a negative regulator of ERK, and further prevent activation of its downstream signaling pathways, including NF- $\kappa B$, thus inhibiting cell viability.

Hint1 increased radiosensitivity in gastric cancer cells. Due to the deleterious influence of Hint1 on cancer cell viability, the present study investigated whether Hintl modulated the radiation resistance of gastric cancer. Two human gastric cancer cell lines with $0,1,2,4$ or 6 Gy X-ray. Prior to the treatment, the protein expression of Hint1 was examined in these cell lines (Fig. 3A). Notably, differences in cell survival were observed between the gastric cancer cells lines following radiation. SGC7901 cells, which demonstrated higher Hint1 protein expression, exhibited higher sensitivity to radiation, 
A

B

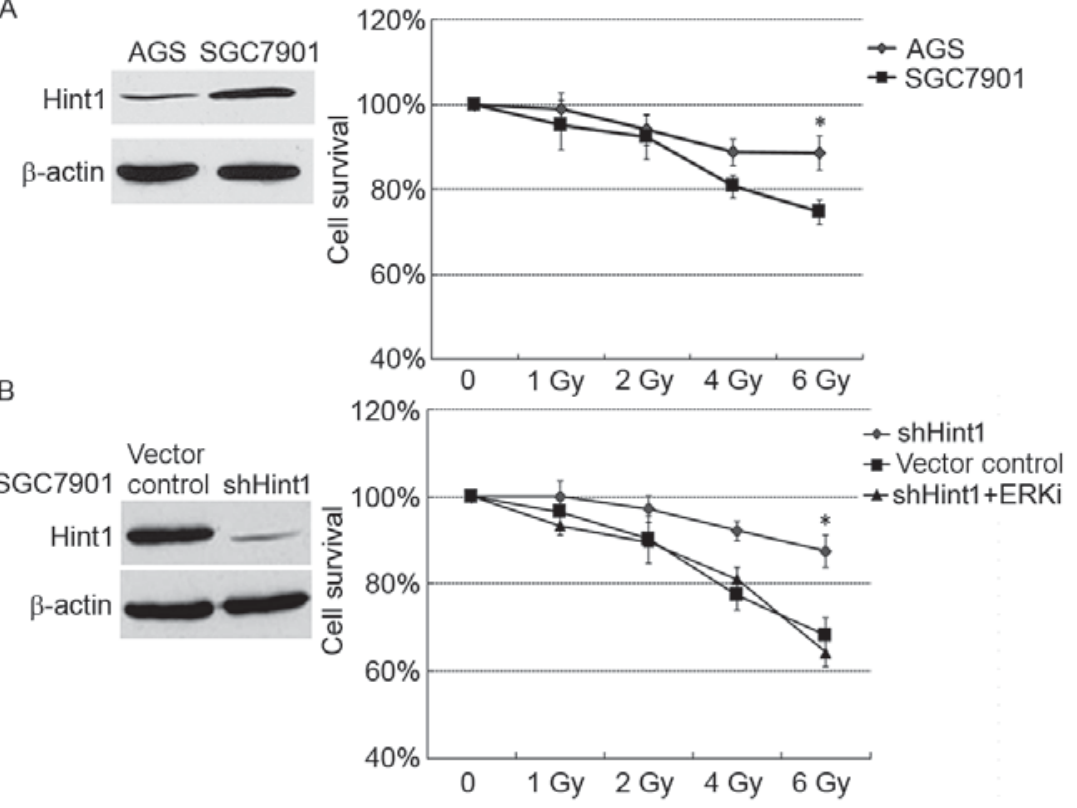

Figure 3. Effect of Hint1 on radiosensitivity. (A) Radiosensitivity was tested by MTT in two gastric cancer cell lines with different Hint1 expression levels. (B) Changes in radiosensitivity induced by Hint1 silencing and ERKi treatment were assessed in SGC7901 cells by MTT assay. Data are presented as the mean \pm standard error of the mean. "P $<0.05$ vs. vector control and shHint1+ERKi groups. Hint1, histidine triad nucleotide-binding protein 1; ERKi, ERK inhibitor; si, small interfering RNA.

A

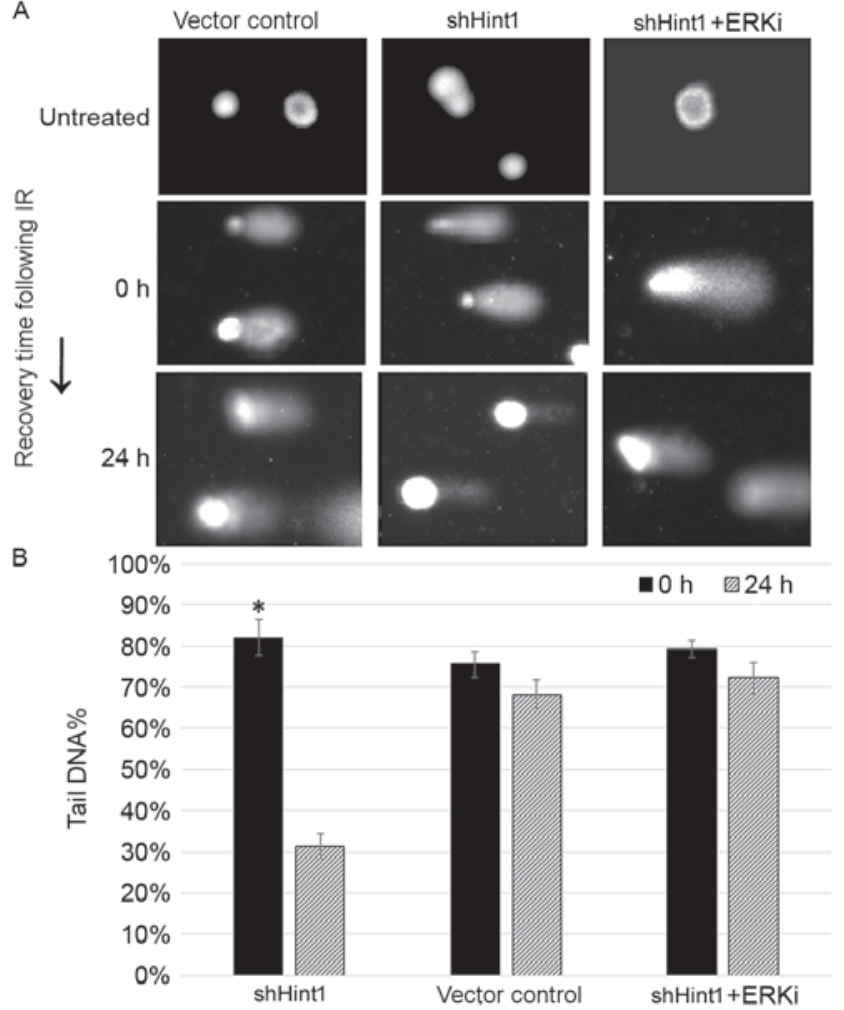

Figure 4. Results of the comet assay. (A) Representative images of comet assay induction prior to and following 6 Gy radiation treatment (magnification, $\mathrm{x} 200)$. (B) The median of DNA in tail (\%) was calculated using Casplab software. Data are presented as the mean \pm standard error of the mean. ${ }^{*} \mathrm{P}<0.05$ vs. 0 h. si, small interfering RNA; Hint1, histidine triad nucleotide-binding protein 1; ERKi, ERK inhibitor.

whereas AGS cells, with lower Hint1 protein expression, were relatively resistant to treatment (Fig. 3A). shHint1-transfected
SGC7901 cells were then treated with radiation, with ERKi or without. Radiation resistance was induced in the shHint1 SGC7901 cells, while ERKi overcame this (Fig. 3B).

Hintl delayed radiation-induced DNA damage repair in gastric cancer cells. A previous study demonstrated that Hint1 is involved in DNA damage repair in normal cells (9). The influence of Hint1 expression on DNA damage, a hallmark of radiation-induced cytotoxicity, was explored on SGC7901 cells using single cell gel electrophoresis, also known as a comet assay. shHint1 cells demonstrated visibly smaller DNA tails than the vector control $24 \mathrm{~h}$ following radiation treatment of $6 \mathrm{~Gy}$, suggesting enhanced DNA repair in Hint1-deficient cells (Fig. 4A). There was no significant difference in the tail DNA fraction between 0 and $24 \mathrm{~h}$ in the vector control and ERKi treatment group (Fig. 4B). In this experiment, ERKi treatment did not affect the formation of DNA damage, instead retarding DNA damage repair.

Hint1-mediated Cyclin D1 suppression results in persistent G1 arrest in gastric cancer cells following IR. The mechanisms underlying the involvement of Hint1 in the radiation response remain unclear. First, the effect of Hint1 expression on the cellular DNA damage response signal pathway was examined. As reported by a previous study, p-ATM levels increase in SGC7901 cells following radiation treatment, but this increase is partially suppressed in shHintl cells (13). Notably, the activation of ATR, the other important DNA damage sensor, did not seem to be altered by Hint1 expression, but it was not possible to explain why Hint1 increased radiosensitivity with enhanced ATM activation (13). Similar results were observed in the present study (Fig. 5A). Therefore, the present study focused on the downstream effectors of the DNA damage response. Cyclin D1, a cell-cycle regulator essential for the G1 phase, was downregulated by Hint1 (Fig. 5B). During the G1 
A
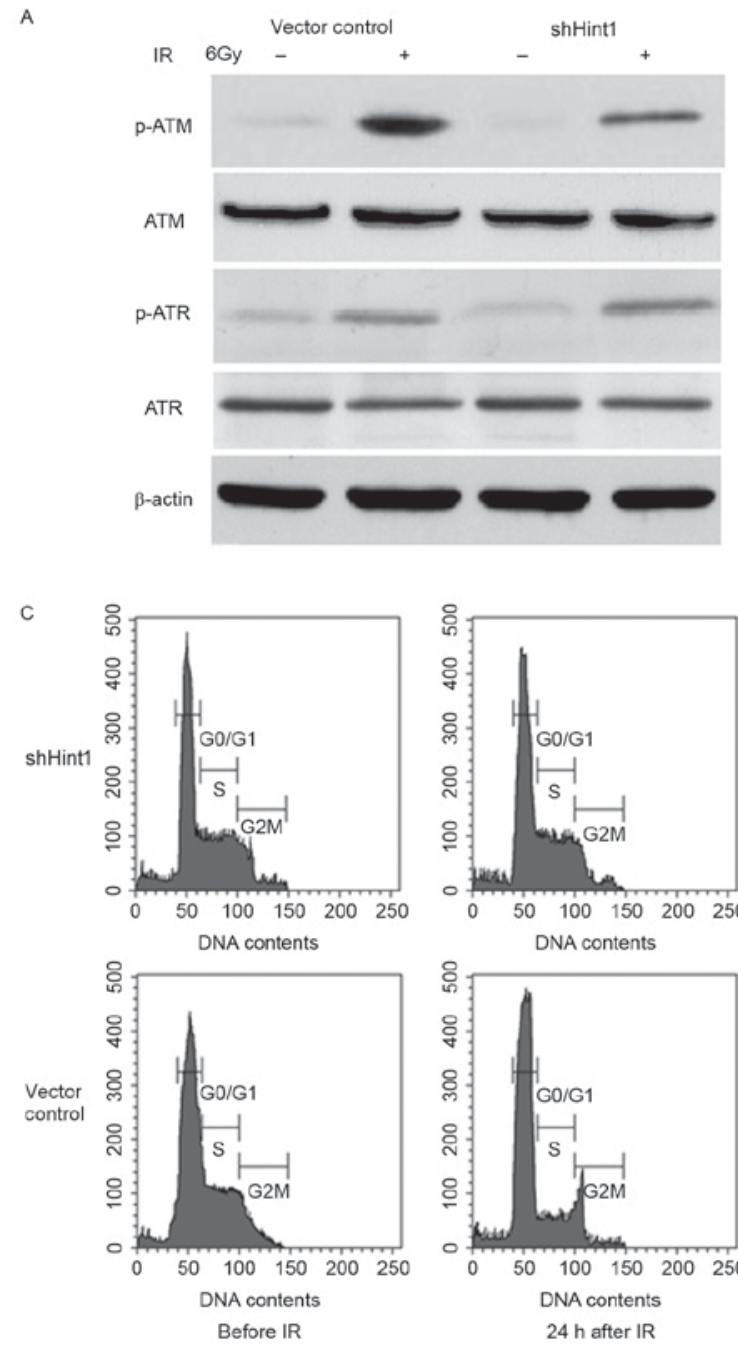
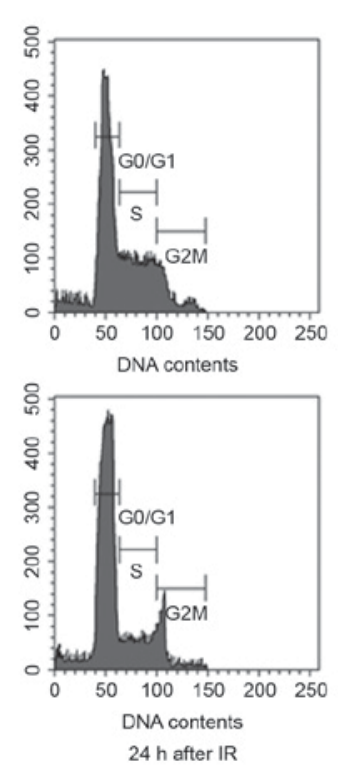
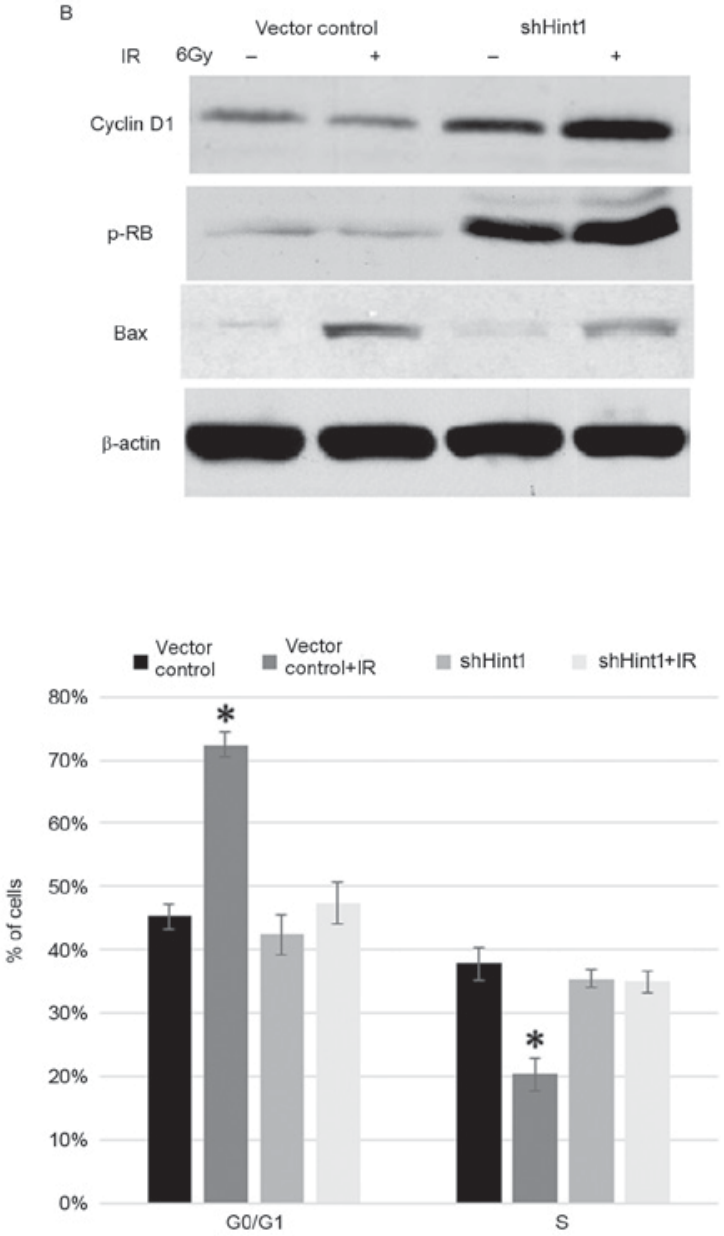

Figure 5. (A) Expression of the DNA damage response sensors, ATM and ATR, was assessed by Western blot analysis. (B) Cyclin D1, p-Rb and Bax protein expression was assessed by Western blot. (C) G1 arrest was observed via flow cytometry in gastric cancer cells following IR. Data are presented as the mean \pm standard error of the mean. "P<0.05 vs. other groups. ATM, ATM serine/threonine kinase; ATR, ATR serine/threonine protein kinase; p-, phosphorylated; Rb, retinoblastoma protein; Bax, BCL2 associated X, apoptosis regulator; IR, ionizing radiation; si, small interfering RNA.

phase, Cyclin D1 increases DNA synthesis via Rb phosphorylation and promotes S phase entry (14). Decreased Cyclin D1 levels in SGC7901 cells significantly inhibited p-Rb activation, resulting in a persistent G1 arrest following IR treatment (Fig. 5B and C). Accordingly, the pro-apoptotic protein Bax was upregulated in vector control cells following IR compared with shHint1 cells following IR (Fig. 5B).

\section{Discussion}

Previous investigations using genetically engineered mice have demonstrated that Hint is a novel haploinsufficient tumor suppressor gene $(2,3)$. It demonstrates a strong analogy with fragile histidine triad, a known tumor suppressor gene on common fragile site fragile site, aphidicolin type, common, fra(3)(p14.2) (2), but its functions are yet to be completely understood. A previous study analyzed clinical samples and reported a potential tumor suppressor role for Hint1 in human gastric cancer (6). In the present study, the function of Hint1 was analyzed in human gastric cancer cells. The results obtained demonstrated, for the first time, that Hintl decreased gastric cancer cell proliferation in vitro by decreasing cell viability. These observations were consistent with the proposed tumor suppressor function of Hint1 and with previous findings demonstrating that Hint1 inhibited human colon and breast cancer cell growth $(2,3,8)$.

Mechanistic studies subsequently demonstrated the negative regulation of Hint1 on ERK signal pathway activation. ERK1/2 are associated protein-serine/threonine kinases that participate in the Ras-Raf-mitogen-activated protein kinase kinase-ERK signal transduction cascade. This cascade participates in the regulation of a number of processes, including cell adhesion, cell cycle progression, cell migration, cell survival, differentiation, metabolism, proliferation and transcription $(11,12)$. ERKs directly phosphorylate multiple transcription factors, including the ETS transcription factor family, c-Jun and c-Myc (15-17). ERK also phosphorylates and activates the $90 \mathrm{kDa}$ ribosomal S6 kinase (p90Rsk), which, in turn, leads to the activation of the transcription factor cAMP response element binding protein (18). Furthermore, ERK results in activation of the $\mathrm{NF}-\kappa \mathrm{B}$ transcription factor by phosphorylating and activating I $\kappa$ B kinase (19). Deregulated activation of $N F-\kappa B$, which 
induces uncontrolled cell growth, is a central signature of multiple types of epithelial cancer. For instance, over-activation of NF- $\kappa \mathrm{B}$, regulated by ERK, promoted malignant transformation in endometrial epithelia cells (20). In the present study, silencing Hint1 resulted in the activation of the ERK-NF- $\kappa \mathrm{B}$ signal pathway in SGC7901 cells. This demonstrated that Hint1 acted as an ERK-control factor in stomach carcinogenesis, resulting in tumor suppression.

As radiation therapy has become a pillar of the therapeutic approach to gastric cancer, the study of the molecular mechanisms underlying the radiation resistance of gastric cancer cells has become important. A previous study indicated that Hint1 $^{-/-}$mouse embryonic fibroblasts cells had defects in the double-stranded break (DSB) repair pathway, including loss of ATM activation and the presence of unrepaired DSB, while another study demonstrated that Hint1-deficient cells exhibited resistance to IR-induced apoptosis $(2,9)$. Therefore, the exact function of Hint1 in response to radiation in gastric cancer cells was worthy of investigation. In the present study, Hint1-deficient SGC7901 cells also exhibited resistance to IR. Although Hint1 deficiency affected ATM activation, DNA damage repair was not retarded in Hint1-deficient SGC7901 cells in the present study. On the contrary, vector control cells demonstrated repressed DNA damage repair functions. One reason for this may be that cancer cells have more inordinate or defective DNA damage repair functions than normal cells. The loss of critical tumor suppressor genes, including Hint1, may activate other pro-survival signal pathways which enhance DNA repair in cancer cells. In addition, ATM activation is not completely inhibited following Hintl silencing or in Hint1 ${ }^{-1}$ cells, as previously described (9). In addition, Hint1 deficiency did not affect the activation of ATR, another important DNA damage sensor, in the present study.

As Hint1 was demonstrated to inhibit ERK activation, its downstream effectors, which may be involved in DNA repair, were investigated. Cyclin D1, a component of the core cell cycle machinery, was revealed to be significantly upregulated following silencing of Hint1. Cyclin D1 phosphorylates and inactivates $\mathrm{Rb}$ protein and promotes progression through the G1-S phase of the cell cycle (21). A number of studies have demonstrated that endogenous high levels of cyclin D1 promote carcinogenesis and radioresistance in the majority of malignant tumors (22-24). Downregulation of Cyclin D1 induces G1 arrest, followed by apoptosis, in human skin cancer A431 cells following UV exposure (25). Cyclin D1 overexpression perturbs DNA replication and induces replication-associated DNA DSBs, which may gradually induce formation of acquired radioresistance during long term fractionated radiotherapy $(26,27)$. In the present study, the negative regulation of ERK mediated by Hint1 may suppress Cyclin D1 expression, which leads to persistent G1 arrest followed by apoptosis in SGC7901 cells following IR exposure.

In summary, the results of the present study first indicated that Hintl was involved in the regulation of cell proliferation and radioresponse via negative control of the ERK signal pathway. These results demonstrated the critical function of Hint in the biology of human gastric cancers. Acting as a tumor growth suppressor and a radiosensitive agent, this protein is a potential biomarker and an attractive target for specific therapeutic interventions against gastric cancer.

\section{Acknowledgements}

The authors would like to thank Dr Hong Fan (Key Laboratory of Developmental Genes and Human Diseases, Ministry of Education, Southeast University, Nanjing, China) for her help and advice.

\section{Funding}

The present study was supported by the National Natural Science Foundation of China (grant no. 81201882), the Natural Science Foundation of Jiangsu Province, China (grant no. BK2012076) and the Key Project supported by the Medical Science and Technology Development Foundation, Nanjing Department of Health, China (grant no. ZKX14039).

\section{Availability of data and materials}

All data generated or analyzed during the present study are included in this published article.

\section{Authors' contributions}

The present study was concieved and designed by XWW and JFC. The methodology was developed by XMW, LZH and ZX. The data was aquired by XWW, JZ, HYZ and XWW. Analysis and interpretation was performed by XMW and XWW. The manuscript was written and revised by XMW and JFC. Administrative and technical support was provided by XWW and ZX. The study was overseen by JFC.

\section{Ethics approval and consent to participate}

The present study was approved by Nanjing Medical University (Nanjing, China) Ethics Committee in 2012.

\section{Consent for publication}

Not applicable.

\section{Competing interests}

The authors declare that they have no competing interests.

\section{References}

1. Stewart BW and Wild CP: World cancer report 2014. World Health Organization. Chapter 1.1 ISBN 9283204298, 2014.

2. Su T, Suzui M, Wang L, Lin CS, Xing WQ and Weinstein IB: Deletion of histidine triad nucleotide-binding protein $1 / \mathrm{PKC}$-interacting protein in mice enhances cell growth and carcinogenesis. Proc Natl Acad Sci USA 100: 7824-7829, 2003.

3. Li H, Zhang Y, Su T, Santella RM and Weinstein IB: Hint1 is a haplo-insufficient tumor suppressor in mice. Oncogene 25: 713-721, 2006

4. Cen B, Li H and Weinstein IB: Histidine triad nucleotide-binding protein 1 up-regulates cellular levels of p27KIP1 by targeting ScfSKP2 ubiquitin ligase and Src. J Biol Chem 284: 5265-5276, 2009.

5. Weiske J and Huber O: The histidine triad protein Hint1 interacts with pontin and reptin and inhibits TCF-beta-catenin-mediated transcription. J Cell Sci 118: 3117-3129, 2005.

6. Huang H, Wei X, Su X, Qiao F, Xu Z, Gu D, Fan H and Chen J: Clinical significance of expression of Hint1 and potential epigenetic mechanism in gastric cancer. Int J Oncol 38: 1557-1564, 2011. 
7. Weiske $\mathrm{J}$ and Huber $\mathrm{O}$ : The histidine triad protein Hint1 triggers apoptosis independent of its enzymatic activity. J Biol Chem 281: 27356-27366, 2006.

8. Wang L, Zhang Y, Li H, Xu Z, Santella RM and Weinstein IB: Hint 1 inhibits growth and activator protein-1 activity in human colon cancer cells. Cancer Res 67: 4700-4708, 2007.

9. Li H, Balajee AS, Su T, Cen B, Hei TK and Weinstein IB: The HINT1 tumor suppressor regulates both gamma-H2AX and ATM in response to DNA damage. J Cell Biol 183: 253-265, 2008.

10. Livak KJ and Schmittgen TD: Analysis of relative gene expression data using real-time quantitative PCR and the 2(-Delta Delta C(T)) method. Methods 25: 402-408, 2001.

11. Samatar AA and Poulikakos PI: Targeting RAS-ERK signalling in cancer: Promises and challenges. Nat Rev Drug Discov 13 928-942, 2014.

12. Huang G, Tang B, Tang K, Dong X, Deng J, Liao L, Liao Z, Yang $\mathrm{H}$ and $\mathrm{He} \mathrm{S}$ : Isoquercitrin inhibits the progression of liver cancer in vivo and in vitro via the MAPK signalling pathway. Oncol Rep 31: 2377-2384, 2014.

13. Smith J, Tho LM, Xu N and Gillespie DA: The ATM-Chk2 and ATR-Chk1 pathways in DNA damage signaling and cancer. Adv Cancer Res 108: 73-112, 2010.

14. Cheng YH, Li LA, Lin P, Cheng LC, Hung CH, Chang NW and Lin C: Baicalein induces G1 arrest in oral cancer cells by enhancing the degradation of cyclin D1 and activating AhR to decrease $\mathrm{Rb}$ phosphorylation. Toxicol Appl Pharmacol 263: 360-367, 2012.

15. Liu H, Duan Z, Zheng H, Hu D, Li M, Tao Y, Bode AM, Dong Z and Cao Y: EBV-encoded LMP1 upregulates Ig $\kappa$ 3'enhancer activity and Igא expression in nasopharyngeal cancer cells by activating the Ets-1 through ERKs signaling. PLoS One 7: e32624, 2012.

16. Park KH, Shin KS, Zhao TT, Park HJ, Lee KE and Lee MK L-DOPA modulates cell viability through the ERK-c-Jun system in PC12 and dopaminergic neuronal cells. Neuropharmacology 101: 87-97, 2016.

17. Kwon YW, Jang S, Paek JS, Lee JW, Cho HJ, Yang HM and Kim HS: E-Ras improves the efficiency of reprogramming by facilitating cell cycle progression through JNK-Sp1 pathway. Stem Cell Res 15: 481-494, 2015.

18. Chuang JI, Huang JY, Tsai SJ, Sun HS, Yang SH, Chuang PC, Huang BM and Ching CH: FGF9-induced changes in cellular redox status and $\mathrm{HO}-1$ upregulation are FGFR-dependent and proceed through both ERK and AKT to induce CREB and Nrf2 activation. Free Radic Biol Med 89: 274-286, 2015.
19. Tsao GSW, Zhu DD, Zhang J and Deng W: Abstract 1046: The role of NF-kB activation in the immortalization of nasopharyngeal epithelial cells. Cancer Res 75, 2015.

20. Mizumoto Y, Kyo S, Kiyono T, Takakura M, Nakamura M, Maida Y, Mori N, Bono Y, Sakurai H and Inoue M: Activation of NF-kappaB is a novel target of KRAS-induced endometrial carcinogenesis. Clin Cancer Res 17: 1341-1350, 2011.

21. Lee Y, Dominy JE, Choi YJ, Jurczak M, Tolliday N, Camporez JP Chim H, Lim JH, Ruan HB, Yang X, et al: Cyclin D1-Cdk4 controls glucose metabolism independently of cell cycle progression. Nature 510: 547-551, 2014.

22. Musgrove EA, Caldon CE, Barraclough J, Stone A and Sutherland RL: Cyclin D as a therapeutic target in cancer. Nat Rev Cancer 11: 558-572, 2011.

23. Peurala E, Koivunen P, Haapasaari KM, Bloigu R and Jukkola-Vuorinen A: The prognostic significance and value of cyclin D1, CDK4 and p16 in human breast cancer. Breast Cancer Res 15: R5, 2013.

24. Choi YJ, Li X, Hydbring P, Sanda T, Stefano J, Christie AL, Signoretti S, Look AT, Kung AL, von Boehmer H and Sicinski P: The requirement for cyclin $\mathrm{D}$ function in tumor maintenance. Cancer Cell 22: 438-451, 2012.

25. Kim AL,AtharM,BickersDR andGautierJ:Ultraviolet-B-induced G1 arrest is mediated by downregulation of cyclin-dependent kinase 4 in transformed keratinocytes lacking functional p53. J Invest Dermatol 118: 818-824, 2002.

26. Shimura T, Ochiai Y, Noma N, Oikawa T, Sano Y and Fukumoto M: Cyclin D1 overexpression perturbs DNA replication and induces replication-associated DNA double-strand breaks in acquired radioresistant cells. Cell Cycle 12: 773-782, 2013.

27. Shimura T, Fukumoto $M$ and Kunugita N: The role of cyclin D1 in response to long-term exposure to ionizing radiation. Cell Cycle 12: 2738-2743, 2013.

This work is licensed under a Creative Commons Attribution-NonCommercial-NoDerivatives 4.0 International (CC BY-NC-ND 4.0) License. 